
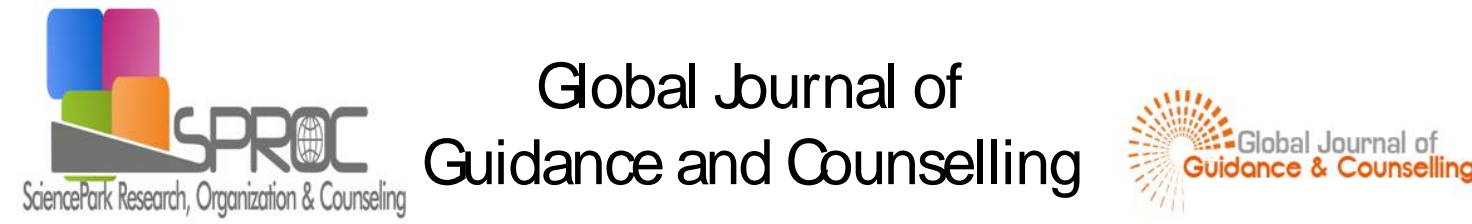

http:// sproc.org/ ojs/ index.php/gigc

\title{
Scale of remembered relationship with parents: Evaluation of psychometric properties on university students
}

Zihniye Okray*

Enil Afsaroglu Eren,

Suggested Citation:

I ck $\quad$ I $\quad c t$

Abstract 
1. Introduction 
l ck $\quad l \quad c t$

\section{Method}

$c t$

\begin{tabular}{lll}
\hline $\mathbf{n}$ & $\%$ \\
\hline
\end{tabular}

Nationality

Gender

Mother

Father

Mother's Educational Level

Father's Educational Level 
3. Data Collection Tools

4. Statistical Analysis 


$\begin{array}{llll}l & c k & l & c t\end{array}$

5. Results

$c t$

$c t$

Scale Total Score

Alienation

Control

$c k$ 
I ck $\quad$ l ct

$\underline{-}$

$\overline{-10}$

,

, 


\section{Discussion}


I ck 
References

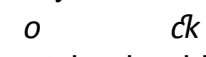

o

${ }_{0} \quad c t \quad{ }_{b} c t$

$\begin{array}{lllllllllllll}M & 0 & 0 & 0 & 0\end{array}$

$c t$

$c t$

$c t$

$c t \quad c t \quad c t$

$c t$ 
I ck

$c k$

o b

o

o ck $c t$

o

$\begin{array}{lll}c t & 0 & c t\end{array}$ 